\title{
Overview of Applications of Laser-Driven Particle Acceleration (Editors Paul R. Bolton, Katia Parodi, and Jörg Schreiber) by CRC Press (Taylor and Francis Group) ISBN 9781498766418 -5 June 2018
}

\author{
Paul R. Bolton * (D), Katia Parodi and Jörg Schreiber \\ Faculty of Physics, Ludwig-Maximilians-Universität München, Am Coulombwall 1, 85748 Garching \\ b. München, Germany; katia.parodi@physik.uni-muenchen.de (K.P.); joerg.schreiber@1mu.de (J.S.) \\ * Correspondence: paul.bolton@physik.uni-muenchen.de; Tel.: +49-89-289-14086
}

Received: 6 September 2018; Accepted: 23 October 2018; Published: 14 November 2018

\begin{abstract}
We briefly describe the new book, Applications of Laser-Driven Particle Acceleration, which was published in June of 2018 by CRC Press, Taylor and Francis Group. Written by the book editors, this article is appropriately an overview. The rationale for developing a book about applications for laser-driven energetic particle and photon sources is given and the content of its two parts (Parts I and II) is described. Part I addresses the current understanding of physical mechanisms and capabilities for acceleration of electrons and ions, as well as for associated generation of energetic photons and neutrons. Part II brings the focus back to potential applications in which the laser-driven source is distinguished from the more fully developed integrated laser-driven accelerator system. For applications of laser-driven sources and systems, a uniqueness strategy is recommended that particularly emphasizes what conventional (not laser-driven) accelerators cannot do. In the context of overall accelerator advancement, this strategy for the laser case highlights the potential for offering new beam configurations and research options that can uniquely augment accelerator capability in the future.
\end{abstract}

\section{Overview}

As a novel component of accelerator advancement, keen interest continues in the development of laser-driven energetic particle and associated photon sources. Understandably, formative studies have focused on candidate source development to optimize distinctive yields from the strong accelerating field in an extreme laser-plasma at a target site or from the longitudinal component of the laser field itself, in a suitably shaped dielectric microstructure. Typical laser power and intensity requirements for electron and ion acceleration in extreme laser-plasma fields mandate a close coupling with high power laser development (up to the multi-petawatt level). It is constructive to distinguish laser-driven source development from integrated laser-driven accelerator system development, with the source as a system component (the ion system example being the integrated laser-driven ion accelerator system (ILDIAS)).

At this embryonic stage, it is critical to establish application categories which include specific applications that are meaningful and doable in the laser-driven case. This can motivate and sustain such novel accelerator development for the longer term, providing practical scientific and technological guidance and justification. Potential applications can also bring essential support for continued high power laser development.

Application requirements can vary significantly, and we anticipate that the integrated laser-driven system will be needed to fulfill those demanding greater sophistication and stability, the realization of which can be longer term. Applications of Laser-Driven Particle Acceleration (ALPA) directly addresses 
potential applications of laser-driven sources and systems [1]. The ALPA book front cover is displayed in Figure 1. Aimed at exposing a broad range of new application potential and at inspiring visionary concepts, this work was approached in two parts.

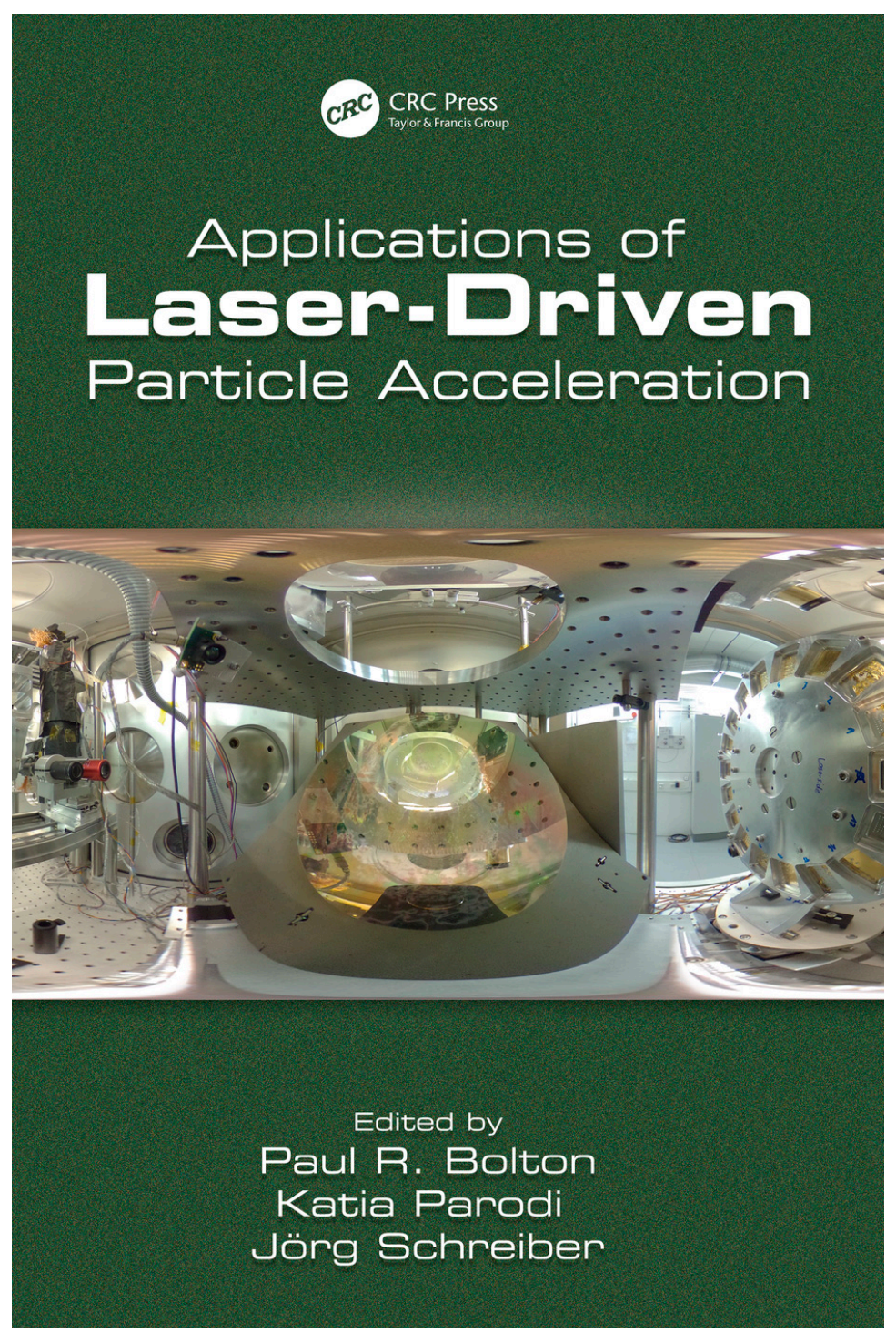

Figure 1. Applications of Laser-Driven Particle Acceleration was published on 5 June 2018 (ISBN 9781498766418). The displayed front cover image is provided by courtesy of CRC Press, Taylor and Francis Group.

Expert contributors of Part I describe our understanding and basic capabilities of electron and ion acceleration, using lasers. This includes electron acceleration by laser-induced plasmas and directly by laser fields in dielectric microstructures. This first part also includes $X$ - and $\gamma$-ray generation associated with laser-driven electron acceleration, as well as energetic neutron generation associated with laser-driven ion acceleration.

A varied assortment of envisioned applications is described in Part II by application experts. This is the core theme of the book for which contributors were encouraged to be visionary. Topics addressed in Part II include: Material studies (ion implantation, ion beam nuclear analysis, dielectric response, and electric discharge comparison), space radiation effects (in electronic devices, such as space solar cells and biological effects), radioisotope production, nuclear reactions in plasmas, fundamental radiobiology and radiation chemistry, laser-driven ion beam radiotherapy, 
particle radiography and imaging, nondestructive elemental assay, and novel neutron sources. These subjects span a broad range of interest, but nonetheless represent a small subset of possibilities. Although feasibility or potential can be more apparent for some applications than for others, we have aimed to motivate a pioneering community of users.

As part of the overall conclusion, ALPA editors emphasize the importance of pursuing a uniqueness strategy for the development and applications of laser-driven energetic sources and systems, which notably should also feature what conventional accelerators (i.e., not laser-driven) are not able to do. The uniqueness of laser-accelerated particle bunches includes, for example, capability for generating bunches of short and ultrashort duration and for generating multiple synchronous particle and photon beams (combining electrons, ions, neutrons, and hard photons in distinctive ways). This strategy also affirms appropriate context by asserting the potential augmentative contribution made by laser-driven particle acceleration to advanced accelerator development.

Applications of Laser-Driven Particle Acceleration is written by laser-driven source experts and many application experts as a useful reference for the scientific and engineering researcher who need not be informed at expert level about relevant laser or laser-plasma physics. Becoming better-informed about the state-of-the-art of laser-driven particle acceleration and some application ideas, the reader can envision new research possibilities. For additional information, the reader can consult the CRC Press website [2]. Further, this book is of particular topical relevance to the Special Issue of this Quantum Beam Science journal, Laser-driven Quantum Beams [3], edited by Paul R. Bolton [4].

Note: The authors of this comment article are editors of the book, Applications of Laser-Driven Particle Acceleration. They are also contributing authors of Chapters 1 (PB, KP and JS), 10 (PB) and 24 (PB, KP, and JS) of this book.

\section{References}

1. Bolton, P.R.; Parodi, K.; Schreiber, J. (Eds.) Applications of Laser-Driven Particle Acceleration; CRC Press: Boca Raton, FL, USA, 2018.

2. Applications of Laser-Driven Particle Acceleration-CRC Press Book. Available online: https: / / www.crcpress.com/Applications-of-Laser-Driven-Particle-Acceleration/Bolton-Parodi-Schreiber/p / book/9781498766418 (accessed on 5 October 2018).

3. QuBS I Special Issue: Laser-driven Quantum Beams. Available online: https://www.mdpi.com/journal/ qubs/special_issues/quantum_beams (accessed on 5 October 2018).

4. Bolton, P.R. Novel Quantum Beams from Integrated Laser-Driven Accelerator Systems. Quantum Beam Sci. 2018, 2, 14. [CrossRef]

(C) 2018 by the authors. Licensee MDPI, Basel, Switzerland. This article is an open access article distributed under the terms and conditions of the Creative Commons Attribution (CC BY) license (http://creativecommons.org/licenses/by/4.0/). 